\title{
INVESTIGACIÓN
}

Recibido: 21/04/2021 --- Aceptado: 03/08/2021 --- Publicado: 08/09/2021

\section{PROYECTOS FORMATIVOS: UNA ALTERNATIVA DIDÁCTICA EN ENTORNO VIRTUAL PARA LA ENSEÑNANA DE LAS CIENCIAS EN MÉXICO}

\author{
Formative project: a didactic alternative in a virtual environment for the \\ teaching of sciences in Mexico
}

iD 8 Juan Salvador Nambo de los Santos': Universidad Pedagógica Nacional/Centro Universitario CIFE. México.

crea-jnambo@upn.mx

\section{Cómo citar el artículo:}

Nambo de los Santos, J. S. (2021). Proyectos formativos: una alternativa didáctica en entorno virtual para la enseñanza de las ciencias en México.Vivat Academia. Revista de Comunicación, 154, 53-69. http://doi.org/10.15178/va.2021.154.e1374

http://www.vivatacademia.net/index.php/vivat/article/view/1374

\section{RESUMEN}

Esta investigación tiene como principal objetivo analizar, bajo el enfoque de la Investigación Acción Educativa, la experiencia de implementar la metodología de Proyectos Formativos en un grupo de Maestría en Enseñanza de las Ciencias de la Universidad Politécnica del Estado de Morelos (Upemor). Los Proyectos Formativos se fundamentan en la teoría educativa de la Socioformación en la cual, resolver problemas del contexto mediante la colaboración y la metacognición es fundamental. Entre las conclusiones se destaca que, los proyectos formativos generan un cambio, tanto en el docente como en el alumno, además de que permiten desarrollar competencias cognitivas fomentando el trabajo colaborativo y la innovación, así como promover una cultura de emprendimiento.

PALABRAS CLAVE: Formación - Proyecto formativo - competencias - método enseñanza - aprendizaje - educomunicación.

\footnotetext{
${ }^{1}$ Juan Salvador Nambo de los Santos: Profesor de la UPN e investigador de CIFE. Autor de artículos y capítulos de libro sobre socioformación, políticas educativas, enseñanza de las ciencias, violencia escolar y libros de texto. Miembro del Sistema Nacional de Investigadores en México, Nivel 1.
} 
Nambo de los Santos, J. S.

Proyectos formativos: una alternativa didáctica en entorno virtual para la enseñanza de las ciencias en México

\section{ABSTRACT}

This article analyses, through Educational Action Research, the experience of implementing the methodology of Formative Projects in a group of Masters in Science Teaching at the Polytechnic University of the State of Morelos (Upemor). The Formative Projects are based on the Socioformation educational theory in which, solving context problems through collaboration and metacognition is fundamental. The findings show that Formative Projects generate a change, both in the teacher and in the student, by promoting the development of cognitive skills, collaborative work, innovation and the culture of entrepreneurship.

KEYWORDS: Formation - project - competences - method - teaching - learning educommunication.

\section{PROJETOS DE FORMAÇÃO: UMA ALTERNATIVA DE ENSINO EM AMBIENTE VIRTUAL PARA O ENSINO DE CIÊNCIAS NO MÉXICO}

\section{RESUMO}

O objetivo principal desta pesquisa é analisar, sob o enfoque da Pesquisa-Ação Educativa, a experiência de implantação da metodologia de Projetos de Formação em um grupo de Mestrado em Ensino de Ciências da Universidade Politécnica do Estado de Morelos (Upemor). Os Projetos de Formação baseiam-se na teoria educacional da Socio Formação em que, resolver problemas do contexto através da colaboração e metacognição é fundamental. Dentro das conclusões, destaca-se que os projetos de formação geram uma mudança, tanto no professor como no aluno, além de permitir o desenvolvimento de competências cognitivas, promovendo o trabalho colaborativo e a inovação, bem como promovendo uma cultura empreendedora.

PALAVRAS CHAVE: Formação - Projeto de formação - competências - método ensino - aprendizagem - educomunicação.

\section{INTRODUCCIÓN}

Ser profesor en cualquier plataforma virtual de aprendizaje implica conocer sobre educación por competencias y sus principales estrategias didácticas, sobre todo una serie de responsabilidades que dan un toque especial al quehacer docente $y$, obviamente, identidad. Las plataformas de aprendizaje en México avanzan de tal manera que, a nivel Iberoamérica, este país es el mayor productor de cursos en línea (Tobón et ál., 2018), cursos cuyos docentes, denominados facilitadores o asesores virtuales incluyen el uso de las redes sociales, simuladores, juegos digitales, podcast, editores de video, entre otros.

Así, no sólo es un sistema de formación en el que se eliminan las barreras de distancia y tiempo, sino que se logran avances que permiten ir más allá del cambio de

Vivat Academia. Revista de Comunicación. 2021, nº 154, 53-69 
Nambo de los Santos, J. S.

Proyectos formativos: una alternativa didáctica en entorno virtual para la enseñanza de las ciencias en México

conductas o cogniciones del alumno (por consecuencia también del docente) y responder a las nuevas necesidades de una sociedad del conocimiento que demanda nuevos procesos de formación (Grosso, 2004). En definitiva, como lo señala MartínCabello: "la ciudadanía global aparece como un recurso retórico importante entre las instituciones globales e incluso entre la ciudadanía, que compra la idea de ser un ciudadano del mundo" (2017, p. 12).

Lo anterior, agregado al contexto de pandemia en el que estamos viviendo en la educación superior en México (Nambo \& Chávez, 2020), representa un desafío importante para una sociedad del conocimiento que busca que las personas trabajen colaborativamente para resolver los problemas en el contexto y mejorar las condiciones de vida con una visión global, compleja y sistémica, teniendo como base las tecnologías de la información y la comunicación (Cabrero et ál., 2020).

\section{OBJETIVOS}

El propósito de este estudio es analizar la experiencia de implementar la metodología de Proyectos Formativos en un grupo de 15 estudiantes de la Maestría en Enseñanza de las Ciencias de la Universidad Politécnica del Estado de Morelos, la cual se realiza de manera semipresencial y en la que se usa Moodle como plataforma virtual de aprendizaje.

En la socioformación, los proyectos formativos consisten en un conjunto articulado de actividades para resolver un problema real del contexto con base en la colaboración, buscando el logro de una serie de propósitos de aprendizaje establecidos en el currículo, junto con la obtención de un producto integrador (Tobón, 2019; Tobón et ál., 2015).

Lo anterior, está vinculado directamente con la transferencia del conocimiento (Dixon, 2001), ya que muestra la preocupación de los maestros por saber si sus alumnos son capaces de resolver problemas reales y si el conocimiento adquirido en la escuela puede ser aplicado para resolver diversas situaciones fuera de ésta.

Los participantes son profesores de Ciencias en distintas instituciones públicas o privadas de la región, quienes reconocían que necesitaban conocimientos en didáctica, planeación y gestión de la información para mejorar su trabajo en clase. Por lo tanto, uno de los principales propósitos fue superar los problemas de la planeación tradicional centrada en contenidos, con el fin de enfocarse en el aprendizaje de los estudiantes.

Cabe señalar que la investigación basada en el diseño de secuencias didácticas es considerada como una línea de investigación emergente en enseñanza de las ciencias (Guisasola et ál., 2021).

Vivat Academia. Revista de Comunicación. 2021, nº 154, 53-69 
Nambo de los Santos, J. S.

Proyectos formativos: una alternativa didáctica en entorno virtual para la enseñanza de las ciencias en México

\section{METODOLOGÍA}

\subsection{Tipo de Estudio}

Se realizó un estudio enmarcado en la investigación-acción educativa (Sandín, 2003), pues se buscó implementar una mejora en la práctica docente. Esto implicaba identificar procesos de metacognición, formación para nuevos referentes educativos, revisión de pares en ciertos momentos con base en la colaboración y socialización de los logros con la comunidad educativa.

\subsection{Procedimiento}

El proceso para aplicar el proyecto formativo Tobón, S. (2019). en los estudiantes de maestría tuvo las siguientes fases:

Fase 1. Dinamización. Sensibilización a los estudiantes sobre los proyectos formativos y su relación con la enseñanza de las ciencias; y planeación de un proyecto formativo a través de una V-heurística.

Fase 2. Desarrollo. Se tomó una secuencia didáctica en el área que a los estudiantes les interesaba y se evaluó mediante un diagrama de flujo para la detección de necesidades. Con base en la evaluación y planeación anteriores se formuló una nueva estructura para la secuencia didáctica, la cual fue la base para su protocolo de investigación, posterior proyecto de tesis y/o publicación de artículo.

Fase 3. Socialización. Foros de discusión para la presentación de proyectos con énfasis en los logros y aspectos a seguir mejorando en el proceso. Presentación de tesis para obtención de grado como Maestros en Enseñanza de las Ciencias.

\subsection{Contexto de la experiencia}

La Universidad Politécnica del Estado de Morelos fue creada en el 2004, atiende a poco más de dos mil alumnos en seis ingenierías, una licenciatura y cuatro maestrías y el modelo educativo establecido desde sus orígenes es el Modelo Basado en Competencias (Upemor, 2021).

La Maestría en Enseñanza de las Ciencias (a partir de ahora MEC) está orientada a la profesionalización docente en las áreas de matemáticas, biología, física y química. Se ofrece de manera semipresencial y cuatrimestral durante dos años. En todo el plan de estudios se incluyen materias relacionadas con la investigación educativa. Los dos primeros cuatrimestres: Investigación educativa I y II. Y los cuatrimestres restantes: Seminario de Investigación I, II, III y IV.

En este artículo se hace énfasis en los dos primeros cuatrimestres que son aquellos relacionados con la presentación de su protocolo de investigación de tesis. Empero, se 
Nambo de los Santos, J. S.

Proyectos formativos: una alternativa didáctica en entorno virtual para la enseñanza de las ciencias en México

les dio seguimiento a todas las materias señaladas como profesor de asignatura responsable, durante la generación 2015-2017:

Tabla 1: Estructura de las dos asignaturas abordadas en la presente experiencia

\begin{tabular}{|c|c|c|}
\hline Asignatura & Investigación educativa 1 & Investigación educativa 2 \\
\hline Justificación & $\begin{array}{l}\text { La asignatura proporcionará a los estudiantes } \\
\text { fundamentos básicos para su investigación } \\
\text { desarrollada en sus actividades académicas } \\
\text { cotidianas con argumento teórico, } \\
\text { fundamentado en estudios realizados por } \\
\text { autores reconocidos. Además de contar con } \\
\text { bases necesarias para precisar los elementos de } \\
\text { la definición del problema }\end{array}$ & $\begin{array}{l}\text { La asignatura proporcionará al } \\
\text { alumno que identifique las } \\
\text { debilidades y fortalezas del } \\
\text { paradigma metodológico e } \\
\text { identifique sus alcances de } \\
\text { investigación educativa }\end{array}$ \\
\hline Objetivo & $\begin{array}{l}\text { Que el alumno desarrolle la capacidad de } \\
\text { identificar, investigar, analizar y estructurar el } \\
\text { planteamiento de un problema }\end{array}$ & $\begin{array}{l}\text { El alumno será capaz de } \\
\text { identificar las características, así } \\
\text { como las particularidades del } \\
\text { paradigma metodológico } \\
\text { cuantitativo, con el fin de valorar } \\
\text { sus fortalezas y debilidades que le } \\
\text { permitan decidir la pertinencia de } \\
\text { su aplicación en el desarrollo del } \\
\text { problema de investigación }\end{array}$ \\
\hline $\begin{array}{l}\text { Unidades de } \\
\text { aprendizaje }\end{array}$ & $\begin{array}{l}\text { 1. Elementos teóricos del planteamiento } \\
\text { de un problema de investigación } \\
\text { 2. Precisar el planteamiento del problema } \\
\text { a investigar, para el desarrollo de la } \\
\text { investigación } \\
\text { 3. Estado del arte de la problemática a } \\
\text { investigar }\end{array}$ & $\begin{array}{l}\text { 1. Paradigma cuantitativo en } \\
\text { investigación educativa } \\
\text { 2. Paradigma cualitativo en } \\
\text { investigación educativa } \\
\text { 3. Marco teórico }\end{array}$ \\
\hline Evaluación & $\begin{array}{l}\text { Actividades del curso: } 80 \% \\
\text { Foros: } 10 \% \\
\text { Trabajo final: } 10 \%\end{array}$ & $\begin{array}{l}\text { Actividades del curso: } 80 \% \\
\text { Foros: } 10 \% \\
\text { Trabajo final: } 10 \%\end{array}$ \\
\hline
\end{tabular}

Fuente: Elaboración propia, 2021

\subsection{Instrumento}

Diagrama de flujo para la detección de necesidades. Este diagrama tiene como propósito autoevaluar el protocolo de investigación, considerando el enfoque socioformativo. Se compone de seis indicadores que valoran la factibilidad del proyecto y el papel que tiene tanto el alumno como el docente (Ver Figura 1).

Vivat Academia. Revista de Comunicación. 2021, nº 154, 53-69 
Nambo de los Santos, J. S.

Proyectos formativos: una alternativa didáctica en entorno virtual para la enseñanza de las ciencias en México

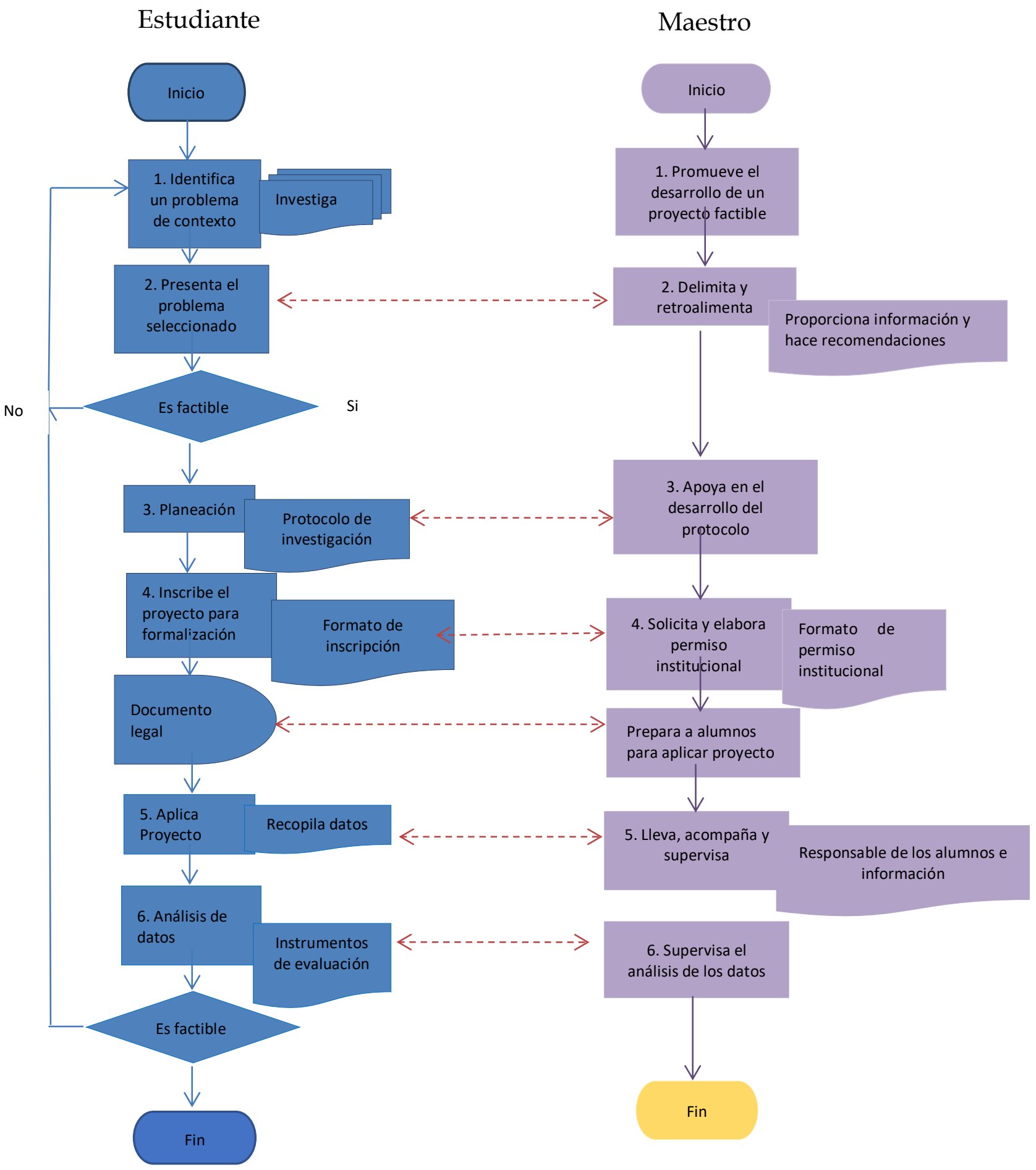

Figura 1: Diagrama de flujo para la detección de necesidades y entrega de protocolo de investigación

Fuente: Elaboración propia basada en Nambo (2019)

La mayoría de las actividades, productos de aprendizaje e instrumentos de evaluación de la MEC estaban contenidos en una plataforma de Moodle administrada por la institución. Cabe destacar que los estudiantes se reunían con el asesor de la 
Nambo de los Santos, J. S.

Proyectos formativos: una alternativa didáctica en entorno virtual para la enseñanza de las ciencias en México

materia dos veces por semestre o en eventos que la institución realizaba de manera específica para ellos. El asesor tenía la libertad de intervenir y adaptar algunos de dichos materiales y dirigir actividades específicas en dichos eventos para enriquecer su trabajo de investigación (Ver Tabla 2).

Tabla 2. Actividades, productos e instrumentos de evaluación durante los dos primeros cuatrimestres

\begin{tabular}{|l|l|l|}
\hline Actividad & Producto de aprendizaje & Instrumento de evaluación \\
\hline $\begin{array}{l}\text { Presentación de proyecto a } \\
\text { través de una V-heurística } \\
\text { (propuesta por el docente) }\end{array}$ & $\begin{array}{l}\text { Mapa conceptual (propuesto } \\
\text { por la plataforma) }\end{array}$ & $\begin{array}{l}\text { Lista de cotejo (propuesto por } \\
\text { la plataforma) }\end{array}$ \\
\hline $\begin{array}{l}\text { Realización de un Estado del } \\
\text { Arte (propuesta por la } \\
\text { plataforma) }\end{array}$ & $\begin{array}{l}\text { Cuadro de doble entrada } \\
\text { (propuesto por la plataforma) }\end{array}$ & $\begin{array}{l}\text { Lista de cotejo (propuesto por } \\
\text { la plataforma) }\end{array}$ \\
\hline $\begin{array}{l}\text { Planeación y ejecución de } \\
\text { proyecto (propuesta por el } \\
\text { docente) }\end{array}$ & $\begin{array}{l}\text { Reporte de resultados } \\
\text { (propuesto por la plataforma) }\end{array}$ & $\begin{array}{l}\text { Lista de cotejo (propuesto por } \\
\text { la plataforma) }\end{array}$ \\
\hline $\begin{array}{l}\text { Avances de reportes de } \\
\text { investigación (propuesta por el } \\
\text { docente) }\end{array}$ & $\begin{array}{l}\text { Presentación electrónica } \\
\text { (propuesto por el docente) }\end{array}$ & $\begin{array}{l}\text { Lista de cotejo (propuesto por } \\
\text { el docente) }\end{array}$ \\
\hline
\end{tabular}

Fuente: Elaboración propia, 2021

\section{RESULTADOS}

\subsection{Fase 1. Dinamización}

En esta fase se logró sensibilizar a los estudiantes sobre los proyectos formativos y su relación con la enseñanza de las ciencias. La mayoría de los profesores-estudiantes tenían como formación inicial la Biología, la Química, las Matemáticas o Ingenierías relacionadas y señalaban tener poca experiencia en el Modelo Basado en Competencias y en la escritura académica.

Como parte de las actividades en la plataforma se le solicitaba al estudiante realizar un trabajo autoadministrado, cumplían con las actividades asincrónicas y el docente tenía la función de generar una retroalimentación asertiva ya sea por los foros de la plataforma o por correo electrónico, WhatsApp e incluso por Skype o de manera presencial. No obstante, para la escritura de los reportes era necesario utilizar la premisa planteada por Ferreiro (2007): Cada época y cada circunstancia histórica da nuevos sentidos a la lectura y a la escritura, especialmente si se considera que en la escuela ocurren diversas cosas que pueden ser contadas de maneras distintas.

Se les conminó a escudriñar en sus archivos, tanto físicos como digitales; en los trabajos de sus alumnos, en las carpetas de evidencia y en sus libreros donde habían guardado sueños, motivaciones y formas de enfrentar la vida. Ellos sabían de literatura especializada. Utilizaban materiales específicos, sitios en YouTube para alimentar sus clases, comenzaban a familiarizarse con juegos, simuladores, realidad aumentada e

Vivat Academia. Revista de Comunicación. 2021, nº 154, 53-69 
Nambo de los Santos, J. S.

Proyectos formativos: una alternativa didáctica en entorno virtual para la enseñanza de las ciencias en México

inteligencia artificial. Sin saberlo eran divulgadores científicos, ya que se encargaban de la alfabetización científica de sus alumnos (Meinardi et ál., 2010). Así que tenían que leer todo de manera distinta. El fin -el propósito- debía ser la escritura (Richardson, 2011).

Los docentes recuperaron las mejores clases que habían tenido, la respuesta a problemáticas emergentes tanto de ellos como de sus estudiantes, así como las vicisitudes de su práctica docente y dieron cuenta de varias experiencias que merecían ser contadas o replicadas. Sin embargo, tenían que ser conscientes de que para escribir era necesario un plan que les ayudara a visualizar el camino a seguir, por lo que las matrices ofrecían una forma de sistematización que favoreció la producción escrita de los docentes (Nambo et ál., 2016). El método era el proyecto formativo. La evidencia inicial: la V-heurística (Ver esquema 2).

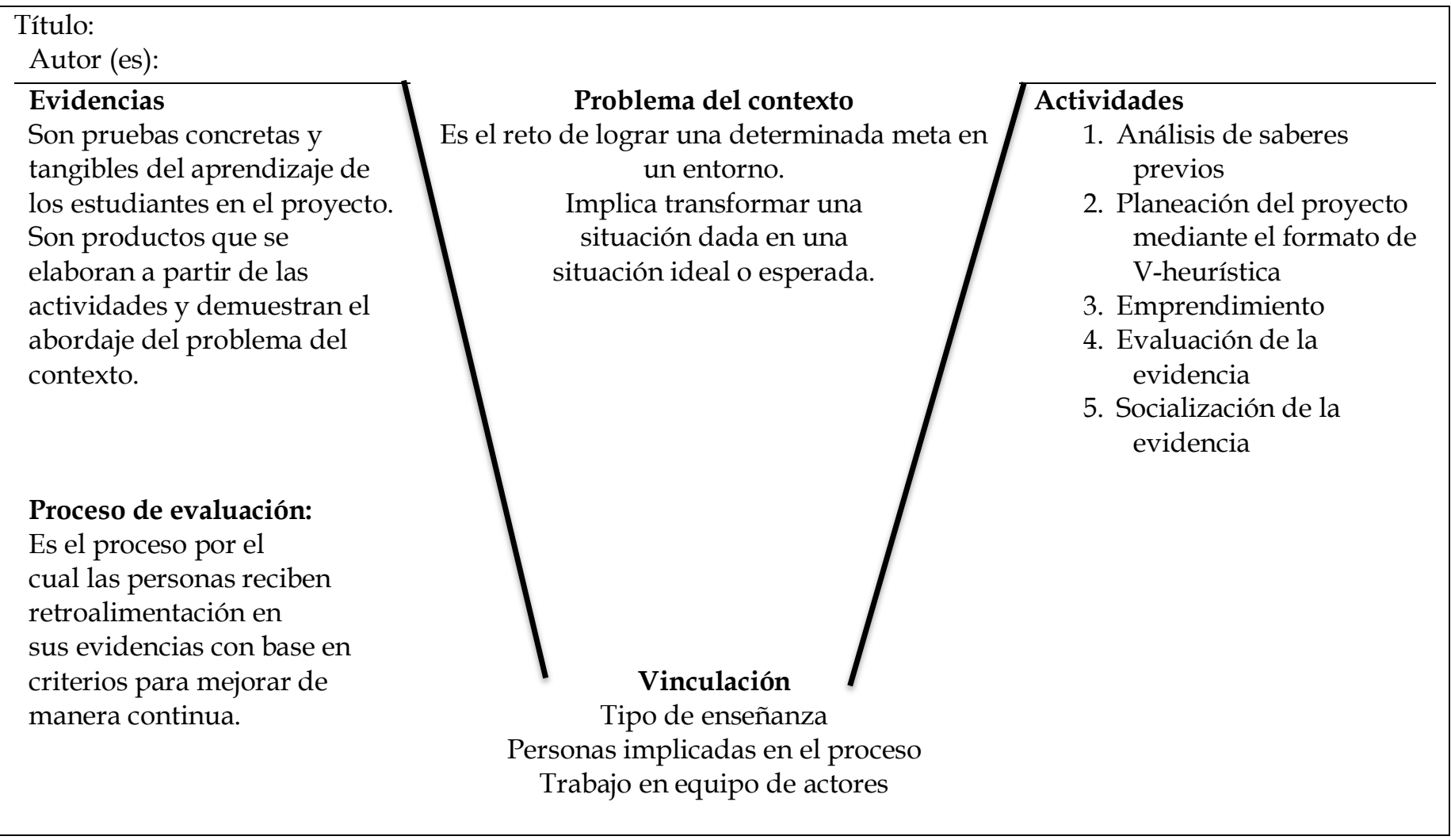

Esquema 2. V-Heurística usada durante las sesiones

Fuente. Elaboración propia. Basado en Tobón (2019)

La evidencia de la V-heurística en este proceso de formación fue de total relevancia, ya que les permitía distinguir el producto concreto que tendrían que presentar como emprendimiento y otros más relacionados. Asimismo, les permitía ver su planeación sin las trabas burocráticas convencionales, como ellos mismos lo expresaban. De esta manera se propusieron proyectos sociales, personales, institucionales, científicos o ambientales. Algunos títulos de proyectos que pasaron por todo el proceso institucional y lograron culminar como tesis fueron los siguientes (Ver Tabla 3): 
Nambo de los Santos, J. S.

Proyectos formativos: una alternativa didáctica en entorno virtual para la enseñanza de las ciencias en México

Tabla 3. Proyectos formativos abordados en el salón de clase

\begin{tabular}{|c|}
\hline Títulos de proyectos \\
\hline $\begin{array}{c}\text { 1. Gestión de la información para el desarrollo de competencias disciplinares en Química } \\
\text { en Enseñanza Media Superior }\end{array}$ \\
\hline $\begin{array}{l}\text { 2. La estrategia del ABP para desarrollar evaluaciones de impacto ambiental desde el } \\
\text { aula }\end{array}$ \\
\hline $\begin{array}{l}\text { 3. La evaluación formativa en la adquisición de las competencias genéricas y } \\
\text { disciplinares en la asignatura de Química Analítica II }\end{array}$ \\
\hline $\begin{array}{l}\text { 4. Estrategia para fomentar la investigación a través del desarrollo de las competencias } \\
\text { disciplinares del área de ciencias experimentales en los alumnos de producción } \\
\text { industrial de alimentos del CBTIS 76. }\end{array}$ \\
\hline $\begin{array}{l}\text { 5. Diseño y aplicación de una estrategia didáctica para generar un aprendizaje } \\
\text { significativo del tema la célula en estudiantes de educación media superior. }\end{array}$ \\
\hline $\begin{array}{c}\text { 6. El desarrollo de competencias para la formación científica de educación secundaria a } \\
\text { través de la aplicación de simuladores en la enseñanza de la física }\end{array}$ \\
\hline
\end{tabular}

Fuente: Elaboración propia, 2021

El proyecto formativo se perfiló como un apoyo para que el profesor sistematizara y desarrollara emprendimientos para trabajar con situaciones emergentes cotidianas que acercaran a los estudiantes al desarrollo de sus capacidades. Dicho proyecto contemplaba seis características importantes:

a) Análisis de saberes previos: El estudiante-docente identificaría los saberes que permiten a sus propios alumnos aprender de la mejor manera, considerando temas pertinentes para la materia; Planeación: Buscaría obtener productos relevantes mediante la planeación y ejecución de actividades y la comunicación de los resultados;

b) Competencias: Contribuiría a formar una o varias competencias relacionadas con los cuatro pilares de la educación (ser, hacer, conocer, convivir);

c) Problemas de contexto: Es el reto de lograr una determinada meta en un entorno, lo cual implicaría transformar una situación dada en una situación ideal o esperada;

d) Emprendimiento: Generaría actividades de relevancia tanto para ellos como para sus estudiantes, relacionados con el mejoramiento del entorno social, individual y medio ambiental;

e) Evaluación: principalmente a través de mapas de aprendizaje, la socialización y la metacognición.

\subsection{Fase 2. Desarrollo}

Una vez planteado el proyecto que los estudiantes de la MEC deseaban realizar y evaluado con el diagrama de flujo para la detección de necesidades y entrega de protocolo de investigación (Ver Figura 1) se formuló una nueva estructura para la secuencia didáctica. Un ejemplo del trabajo realizado es el siguiente: 
Nambo de los Santos, J. S.

Proyectos formativos: una alternativa didáctica en entorno virtual para la enseñanza de las ciencias en México

Se revisó la metodología de las secuencias didácticas en un Colegio de Bachilleres del estado de Morelos, a la cual estaba adscrito el estudiante de maestría y se implementó la metodología de proyectos formativos.

En la Tabla 5 se presenta el formato elaborado para la nueva propuesta de planeación, que busca aplicar los referentes de la socioformación y se caracteriza por los siguientes elementos:

1) se enfoca en los aprendizajes esperados como eje clave, en lugar de propósitos y objetivos, que es una de las confusiones recurrentes entre los docentes que no tienen una formación docente previa;

2) los aprendizajes esperados se sintetizan para que sean comprensibles y se les solicita que trabajen como máximo dos de ellos;

3) se incorpora la resolución de problemas del contexto, de tal manera que se supere el énfasis en contenidos;

4) énfasis en el abordaje de actividades dinámicas y motivantes con los estudiantes; $y$

5) tener un formato más sencillo de planeación que sirva de guía a los estudiantes más que ser un instrumento administrativo o de control de actividades. 
Nambo de los Santos, J. S.

Proyectos formativos: una alternativa didáctica en entorno virtual para la enseñanza de las ciencias en México

Tabla 4. Mejora en la planeación del proyecto formativo respecto a su componente didáctico (ejemplo)

\begin{tabular}{|c|c|}
\hline $\begin{array}{l}\text { Título del proyecto } \\
\text { formativo }\end{array}$ & $\begin{array}{l}\text { Gestión de la Información para la Enseñanza de la Química en } \\
\text { Educación Media Superior }\end{array}$ \\
\hline Asignatura (s) & Química I \\
\hline $\begin{array}{l}\text { Reto o problema del } \\
\text { contexto que deben abordar } \\
\text { o resolver los estudiantes }\end{array}$ & $\begin{array}{l}\text { Aplicar la gestión de la información de videos y otras herramientas } \\
\text { y/o estrategias didácticas para despertar en el estudiante el interés por } \\
\text { el aprendizaje de la Química, lo que le permita valorar sus aplicaciones } \\
\text { en diferentes contextos e involucrarlo en la experiencia estimulante y } \\
\text { satisfactoria de aprender y estudiar }\end{array}$ \\
\hline $\begin{array}{l}\text { Producto central para } \\
\text { lograr en todo el proyecto } \\
\text { formativo por parte de los } \\
\text { estudiantes a partir de la } \\
\text { resolución del problema }\end{array}$ & $\begin{array}{l}\text { Líneas de tiempo a partir del uso de videos para la enseñanza de la } \\
\text { química }\end{array}$ \\
\hline \multicolumn{2}{|c|}{ Contenidos mínimos (saberes esenciales) } \\
\hline Fases & Actividades \\
\hline Fase de apertura & $\begin{array}{l}\text { Se presenta el contenido general y forma de evaluación de la } \\
\text { asignatura, con la finalidad que los estudiantes se familiaricen con la } \\
\text { misma. Se enfatiza en los productos que los estudiantes deben entregar } \\
\text { a lo largo del curso, indicando que se conformará un portafolio de } \\
\text { evidencias individual. Material Didáctico: Cuestionario impreso, } \\
\text { pizarrón, marcador para pizarrón, lápiz o lapicero, bolígrafo, video } \\
\text { número 1. }\end{array}$ \\
\hline Fase de desarrollo & $\begin{array}{l}\text { Se lleva a cabo una evaluación diagnóstica, se utilizan } 11 \text { videos de } \\
\text { YouTube para un bloque, de estos solo el primero se utilizó para } \\
\text { comprender el concepto de química su desarrollo histórico y su } \\
\text { relación con otras ciencias; dependiendo del número total de } \\
\text { estudiantes, en el grupo se integraron equipos homogéneos pudiendo } \\
\text { ser estos de un mínimo de } 4 \text { integrantes para la observación de videos } \\
\text { y en este, la elaboración de una línea de tiempo sobre la química con } \\
\text { los principales momentos de su desarrollo y mostrar los momentos } \\
\text { trascendentales que ha vivido esta ciencia en el ámbito nacional e } \\
\text { internacional y el contexto histórico y social en el que surge. } \\
\text { El estudiante elaboró en el aula y por equipos de } 2 \text { personas una línea } \\
\text { de tiempo, en tanto recibió asesoría por parte del maestro. }\end{array}$ \\
\hline Fase de cierre & $\begin{array}{l}\text { Exposición al grupo de la línea de tiempo. Para la evaluación de esta } \\
\text { actividad se elaboró una rúbrica (Ver Tabla 1), con esta rúbrica } \\
\text { también se evaluarán los reportes de los videos, sin } \\
\text { tomar en cuenta el punto de la "postura". } \\
\text { Se analizó con los estudiantes las conclusiones obtenidas sobre los } \\
\text { productos. Evidencia: Línea de tiempo }\end{array}$ \\
\hline
\end{tabular}

Fuente: Elaboración propia a partir de Vargas et ál. (2019)

\subsection{Fase 3. Socialización}

El ejemplo anterior forma parte de un artículo que fue realizado por parte de un estudiante de la MEC en coautoría con sus directores de tesis. La propuesta logró que sus alumnos mejoraran su rendimiento académico, por lo que dicha propuesta se replicó en otros grupos y otras instituciones pertenecientes al Colegio de Bachilleres. 
La gestión de la información que en primera instancia realizó el docente sirvió para identificar sitios valiosos en Youtube para la enseñanza de la química. El proyecto que se inició desde los primeros cuatrimestres logró concretarse en un aporte al estado del conocimiento, el cual representó una de las mejores formas de socializar los resultados de cada uno de los proyectos formativos. Asimismo, se desarrollaron foros de discusión que les permitía a los estudiantes de maestría retroalimentación externa para sus proyectos.

Cabe destacar que la planeación didáctica basada en proyectos propuesta en la Fase 2 , sirvió también como base para el asesor de los estudiantes de maestría para elaborar su propio proyecto, articulando las asignaturas de la Tabla 1.

Lo anterior se hizo para abordar el tema de la escritura y el desarrollo de proyectos a partir de su propia experiencia docente. En esta nueva propuesta, la evaluación se centra en lograr un producto integrador pertinente al entorno y motivante para los estudiantes, que posibilite valorar los aprendizajes esperados de ambas asignaturas y la detección de necesidades y entrega de protocolo de investigación (ver Tabla 5). 
Nambo de los Santos, J. S.

Proyectos formativos: una alternativa didáctica en entorno virtual para la enseñanza de las ciencias en México

Tabla 5. Mejora en la planeación del proyecto formativo respecto a su componente didáctico

\begin{tabular}{|c|c|}
\hline $\begin{array}{l}\text { Título del proyecto } \\
\text { formativo }\end{array}$ & $\begin{array}{l}\text { Atrévete a escribir: Iniciación a la escritura de tu proyecto de } \\
\text { investigación }\end{array}$ \\
\hline Asignatura (s) & Investigación educativa I y II \\
\hline $\begin{array}{l}\text { Reto o problema del } \\
\text { contexto que deben } \\
\text { abordar o resolver los } \\
\text { estudiantes }\end{array}$ & $\begin{array}{l}\text { Generar ambientes de aprendizaje que permitan a los participantes la } \\
\text { apropiación de herramientas teórico-conceptuales y metodológicas, para } \\
\text { avanzar en su proyecto de investigación. }\end{array}$ \\
\hline $\begin{array}{l}\text { Producto central para } \\
\text { lograr en todo el } \\
\text { proyecto formativo } \\
\text { por parte de los } \\
\text { estudiantes a partir de } \\
\text { la resolución del } \\
\text { problema }\end{array}$ & Protocolo de investigación \\
\hline \multicolumn{2}{|c|}{ Contenidos mínimos (saberes esenciales) } \\
\hline Fases & Actividades \\
\hline Fase de apertura & $\begin{array}{l}\text { Sensibilizar sobre el proceso de lectura y escritura como parte de su } \\
\text { proyecto ético de vida. } \\
\text { Analizar lecturas especializadas que permitan al estudiante avanzar en } \\
\text { su estado del arte. } \\
\text { Señalar mitos en torno al proceso de escritura. } \\
\text { Identificar fuentes de escritura que permitan desarrollar el proyecto } \\
\text { académico laboral del estudiante (fotografías, diario de campo, bitácora, } \\
\text { registro de actividades). } \\
\text { Propiciar ejercicios que permitan vincular los proyectos académicos } \\
\text { laborales de los estudiantes con su formación en la MEC } \\
\text { Realizar una V-heurística con las temáticas de su proyecto de } \\
\text { investigación }\end{array}$ \\
\hline Fase de desarrollo & $\begin{array}{l}\text { Identificar reglas generales de ortografía, redacción, búsqueda } \\
\text { especializada de información, uso de fotografías y citado que establece el } \\
\text { sistema APA. } \\
\text { Desarrollar un planteamiento basado en el modelo de proyectos } \\
\text { formativos, la indización y un cronograma de actividades. } \\
\text { Plantear estrategias para evitar los diferentes conflictos en el proceso de } \\
\text { escritura (normas de ortografía, redacción, uso de tecnicismos). } \\
\text { Revisión y adaptación de sus secuencias didácticas } \\
\text { Foros presenciales de presentación de avances de tesis }\end{array}$ \\
\hline Fase de cierre & $\begin{array}{l}\text { Elaboración de un planteamiento de problema. } \\
\text { Desarrollar un proyecto bajo el enfoque socioformativo. } \\
\text { Identificar las principales competencias a desarrollar con su proyecto } \\
\text { académico laboral. } \\
\text { Entrega del protocolo de investigación. } \\
\text { Foros presenciales de presentación de avances de tesis }\end{array}$ \\
\hline
\end{tabular}

Fuente: Elaboración propia 
Nambo de los Santos, J. S.

Proyectos formativos: una alternativa didáctica en entorno virtual para la enseñanza de las ciencias en México

\section{DISCUSIÓN}

Barber y Mourshed (2008) afirman que las experiencias de éxito de 25 sistemas educativos resaltan tres puntos por atender e incidir en su desempeño de manera positiva: conseguir a las personas más aptas para ejercer la docencia; desarrollarlas hasta convertirlas en instructores eficientes; y garantizar que el sistema sea capaz de brindar la mejor instrucción posible. Esto sigue valorándose en México, donde la enseñanza de las ciencias sigue siendo un reto en todos sus niveles.

Los docentes encargados de la enseñanza de las ciencias en México cuentan con un perfil distinto al de los profesores normalistas, pero que se va construyendo bajo una perspectiva enriquecida con los saberes de la experiencia y que sitúa al docente como voz autorizada de la sociedad del conocimiento.

Ante dicha situación las secuencias de enseñanza-aprendizaje se convierten en una herramienta indispensable tanto para el trabajo presencial como a través de las plataformas virtuales de aprendizaje. En este sentido, la pandemia causada por Covid 19 ha difuminado las fronteras y las identidades que se mantienen en torno a la profesión docente, especialmente respecto al uso de herramientas digitales.

En este estudio se presenta una propuesta sencilla centrada en las actividades clave que se propone realizar, a partir de la resolución de un problema del entorno. Las planeaciones didácticas usadas por los estudiantes de maestría tenían una gran cantidad de componentes que dificultaban su realización y terminaban siendo una actividad más administrativa que enfocada en el aprendizaje de sus alumnos.

\section{CONCLUSIONES}

Al término del Proyecto Formativo que se promovió en este proyecto de investigación-acción, los estudiantes-docentes comentaron que encontraban mayor aplicación de los conocimientos contenidos en su materia.

En este sentido, los estudiantes afirmaban tener la capacidad de estudiar por motivación para resolver un problema de su propio contexto, además de que les permitía desarrollar competencias tanto para ellos como para sus estudiantes, fomentando la innovación, así como promover una cultura de emprendimiento y la educomunicación (Watson, 2001; Aramburuzabala, ét al, 2013; y Jiménez-Marín ét al, 2020; Elias-Zambrano, ét al, 2021).

Implementar la metodología de proyectos formativos tanto de manera particular como para los estudiantes permitió mayor autonomía y menos estrés, ya que había más claridad de lo que se pretendía lograr y de la manera como se articulaban las evidencias tanto de la Maestría como de sus clases de Ciencias.

Cabe mencionar que se llevó a cabo una evaluación crítica de la metodología de las secuencias didácticas en las instituciones en las que los estudiantes laboraban, donde

Vivat Academia. Revista de Comunicación. 2021, nº 154, 53-69 
Nambo de los Santos, J. S.

Proyectos formativos: una alternativa didáctica en entorno virtual para la enseñanza de las ciencias en México

se observó la falta de abordaje de problemas reales del contexto. Esto es común en muchas propuestas de secuencias didácticas, que, aunque pretenden ser significativas, se quedan en el abordaje de preguntas conceptuales.

\section{REFERENCIAS}

Aramburuzabala, P., Hernández-Castilla, R., Ángel - Uribe, I. C. (2013) Modelos y tendencias de la formación docente universitaria Profesorado. Revista de Currículum y Formación de Profesorado, 17(3), 345-357.

Barber, M., y Mourshed, M. (2008). Cómo hicieron los sistemas educativos con mejor desempeño del mundo para alcanzar sus objetivos. PREAL.

Cabrero, E., Carreón, V. y Guajardo, M. (2020). México frente a la sociedad del conocimiento. La difícil transición. CIDE, Siglo XXI editores.

Dixon, N. (2001). El conocimiento común. Cómo prosperan las compañías que comparten lo que saben. Oxford University Press.

Ferreiro, E. (2007). Leer y escribir en un mundo cambiante. Versión. Estudios de Comunicación y Política, 11, 99-112.

Guisasola, J., Ametller, J., y Zuza, K. (2021) Investigación basada en el diseño de Secuencias de Enseñanza-Aprendizaje: una línea de investigación emergente en Enseñanza de las Ciencias. Revista Eureka sobre Enseñanza y Divulgación de las Ciencias, 18, 1-18. https://doi:10.25267/rev_eureka_ensen_divulg_cienc.2021.v18.i1.1801

Grosso, J. L. (2004). NTICS, educación virtual, lógicas de la comunicación social y "sociedad del conocimiento": anotaciones sobre el contexto y la responsabilidad social de la educación superior a distancia en ALC. Revista Diálogo Educacional, 4(13), 69-78.

Jiménez-Marín, G., Elías Zambrano, R. y Bellido-Pérez, E. (2020). 'Meetings with Professionals': The Success of An Innovative Teaching Methodology Based on Real Business Scenarios at the University of Seville, Spain. International Journal of Social Policy and Education, 2 (8), 12-20.

Martín-Cabello, A. (2017). Ciudadanía global. Un estudio sobre las identidades sociopolíticas en un mundo interconectado. Arbor, 193(786), a422-a422. http://dx.doi.org/10.3989/arbor.2017.786n4010

Meinardi, E., González, L., Revel, A., \& Plaza, V. (2010). Educar en ciencias. Paidós. 
Nambo de los Santos, J. S.

Proyectos formativos: una alternativa didáctica en entorno virtual para la enseñanza de las ciencias en México

Nambo de los Santos, J. S. (2019). Investigación-acción: estrategia para la sustentabilidad. En Frías, G. y Montes, L. (Coord.). Arar, sembrar y cosechar: planteles educativos sustentables (pp. 104-108). UAEM

Nambo de los Santos, J. S., \& Chavez, V. G. (2020). Conceptual Cartography of School Safety: School Experiences in Managing the Covid-19 Pandemic in Mexico. Revista Romaneasca pentru Educatie Multidimensionala, 12(2Sup1), 86-92. https://doi.org/10.18662/rrem/12.2sup1/293

Nambo de los Santos, J. S., Rodríguez, E. y Tobón, S. (2016). Proyectos formativos y análisis de casos en el desarrollo de la competencia de escritura. En Tobón, S., Guzmán, C., Vélez, J. y Nambo de los Santos, J. S. (Coord.). Socioformación y sociedad del conocimiento. Experiencias en organizaciones empresariales, educativas y comunitarias (pp. 257-274). Kresearch.

Richardson, R. (2011). Primero leemos, después escribimos. El proceso creativo según Emerson. FCE.

Sandín Esteban, M. P. (2003). Investigación cualitativa en Educación. Fundamentos y Tradiciones. Mc Graw Hill.

Tobón, B., Tobón, S., Veytia-Bucheli, M. G., \& Escudero, A. (2018). Hacia un nuevo concepto: Plataformas Virtuales Socioformativas (PVS). Revista Espacios, 39(53), 2727.

Tobón, S. (2019). Metodología de los proyectos formativos._ Kresearch.

Tobón, S., González, L., Nambo de los Santos, J. S., \& Vázquez Antonio, J. M. (2015). La Socioformación: Un Estudio Conceptual. Paradígma, 36(1), 7-29.

Vargas, B., Zapata, C. y Nambo de los Santos, J. S. (2019). Gestión de la Información para la Enseñanza de la Química en Educación Media Superior. Academicus. 1(15), 58-69.

Watson, D. M. (2001). Pedagogy before technology: rethinking the relationship between ICT and teaching. Education and Information Technologíes, 6 (4). 251-266.

Upemor. (2021). Universidad Politécnica del Estado de Morelos. https://www.upemor.edu.mx/

Elias-Zambrano, R., Sánchez-Gey, N., Román-San-Miguel, A. (2021). La evolución del aprendizaje a través de los medios: publicidad, comunicación audiovisual y periodismo como base de la educomunicación. En La comunicación a la vanguardia. Tendencias, métodos y perspectivas, 1664-1675. 
Nambo de los Santos, J. S.

Proyectos formativos: una alternativa didáctica en entorno virtual para la enseñanza de las ciencias en México

\section{AUTOR/ES:}

Juan Salvador Nambo de los Santos:

Doctor en Educación. Integrante del Sistema Nacional de Investigadores en México (SNI 1). Posdoctorado en Metodología de la Investigación Científica, Socioformación y Desarrollo Humano. Adscrito a la línea de investigación: Socioformación y Educación para la ciudadanía global. Autor de artículos y capítulos de libro sobre socioformación, políticas educativas, enseñanza de las ciencias y libros de texto. Actualmente se encuentra adscrito al Centro Universitario CIFE, a la Universidad Pedagógica Nacional y al Centro de Estudios Superiores "Lic. Benito Juárez García”. salvadornambo@gmail.com

ORCID iD: https:// orcid.org/0000-0002-3891-5228

Researcher ID Thomson: https:// publons.com/researcher/AAR-7717-2020/

Google Scholar: https://scholar.google.es/citations?user=ncS5l3EAAAAJ\&hl=es

ResearchGate: https://www.researchgate.net/profile/Juan-Nambo

Academia.edu: https://upn.academia.edu/JuanNambo 